
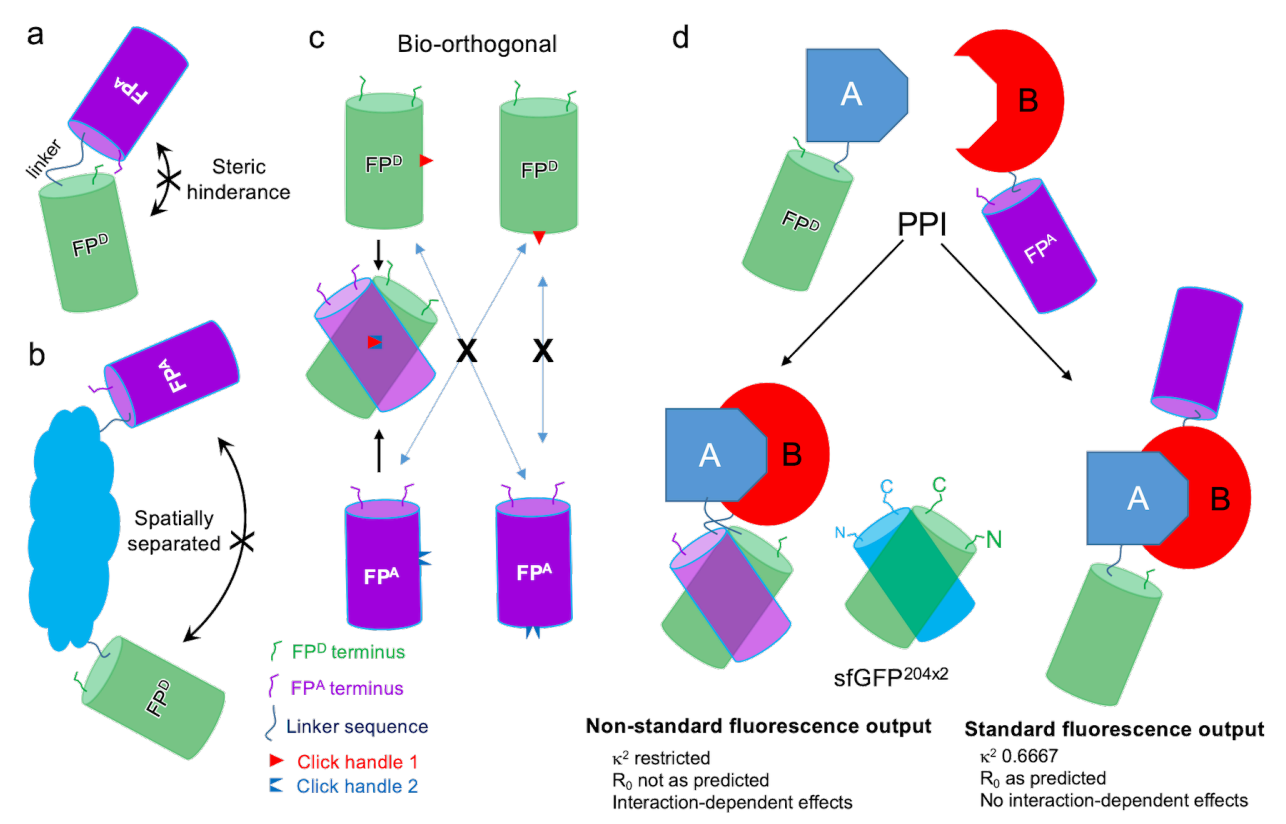

\title{
The effect of proximity on the function and energy transfer capability of fluorescent protein pairs.
}

Jacob R. Pope ${ }^{1}$, Rachel L. Johnson ${ }^{1}$, W. David Jamieson ${ }^{3}$, Harley L Worthy ${ }^{1, a}$, Senthilkumar D. Kailasam ${ }^{5,6}$, Husam Sabah Auhim ${ }^{1,4}$, Daniel W. Watkins ${ }^{1, b}$, Pierre Rizkallah$^{2}$, Oliver Castell ${ }^{3}$, D. Dafydd Jones ${ }^{1 *}$.

1. Molecular Biosciences, School of Biosciences, Cardiff University, Cardiff, UK.

2. School of Medicine, Cardiff University, Cardiff, UK.

3. School of Pharmacy, Cardiff University, Cardiff, UK.

4. Department of Biology, College of Science, Baghdad University, Baghdad, Iraq.

5. McGill University and Genome Quebec Innovation Centre, Montreal, QC, Canada,

6. Department of Human Genetics, McGill University, Montreal, QC, Canada

* Corresponding author. D. Dafydd Jones, Molecular Biosciences Division, School of Biosciences, Cardiff University, Cardiff, UK. CF10 3AT. Email: jonesdd@cardiff.ac.uk. Tel: +442920874290.

Current address:

${ }^{a}$ Henry Wellcome Building for Biocatalysis, Biosciences, University of Exeter, Exeter, United Kingdom

${ }^{\mathrm{b}}$ School of Biochemistry, University of Bristol, Bristol, United Kingdom. 


\section{Abstract}

Fluorescent proteins (FPs) are commonly used in pairs to monitor dynamic biomolecular events through changes in their proximity via distance dependent processes such as Förster resonance energy transfer (FRET). Many FPs have a tendency to oligomerise, which is likely to be promoted through attachment to associating proteins through increases in local FP concentration. We show here that on association of FP pairs, the inherent function of the FPs can alter. Artificial dimers were constructed using a bioorthogonal Click chemistry approach that combined a commonly used green fluorescent protein (superfolder GFP) with itself, a yellow FP (Venus) or a red FP (mCherry). In each case dimerisation changes the inherent fluorescent properties, including FRET capability. The GFP homodimer demonstrated synergistic behaviour with the dimer being brighter than the sum of the two monomers. The structure of the GFP homodimer revealed that a water-rich interface is formed between the two monomers, with the chromophores being in close proximity with favourable transition dipole alignments. Dimerisation of GFP with Venus results in a complex displaying $~ 86 \%$ FRET efficiency, which is significantly below the near $100 \%$ efficiency predicted. When GFP is complexed with mCherry, FRET and mCherry fluorescence itself is essentially lost. Thus, the simple assumptions used when monitoring interactions between proteins via FP FRET may not always hold true, especially under conditions whereby the protein-protein interactions promote FP interaction. 


\section{Introduction}

Fluorescent proteins (FPs) have revolutionised biology through their use as genetically encoded imaging tags and biosensors ${ }^{1-6}$. The subsequent engineering of a small subset of natural FPs ${ }^{1,2}$, especially green fluorescent protein (GFP) from Aequorea victoria ${ }^{7}$ and DsRed from coral ${ }^{5}$ have expanded their use by changing their spectral (e.g. $\lambda_{\max }, \lambda_{\mathrm{EM}}$, quantum yield, brightness) and structural (e.g. quaternary structure, stability, folding kinetics, chromophore maturation kinetics) properties. One of the most important uses of FPs is to monitor dynamic biological events such as protein-protein interactions using processes such as Förster resonance energy transfer (FRET) ${ }^{8,9}$. FRET is largely a passive process that relies on two FPs with mutually compatible spectral properties (acceptor FP absorbance overlapping with donor FP emission wavelength) being in close proximity; changes in distance between the two FPs changes efficiency of FRET between the donor and acceptor.

Despite FRET being a mainstay of biomolecular interaction analysis, there are a several assumptions required such as freely rotating FPs that do not interact or align in any significant manner. As well as absolute distance between the FPs, the angular vector between the chromophore dipoles is critical; this is the $\kappa^{2}$ value equation 1 .

$$
R_{0}=0.211 \sqrt[6]{\mathrm{k}^{2} n^{-4} Q_{D} J(\mathrm{l})} \quad \text { Equation } 1
$$

where $R_{0}$ is the Förster radius, $\kappa^{2}$ is the dipole orientation factor, $n$ is the solvent refractive index, $Q_{D}$ is the quantum yield of the donor and $J(\lambda)$ is the overlap integral between the donor emission and acceptor molar absorbance. $R_{0}$ is used as a constant to relate energy transfer efficiency to distance between individual components via equation 2.

$r=R_{0} \sqrt[6]{(1-E) / E} \quad$ Equation 2

where $r$ is the distance between two FRET chromophores and $E$ is the observed FRET efficiency. Critically $\kappa^{2}$ is arbitrarily set to 0.667 to reflect two randomly orientated chromophores as the dipole orientation is largely unknown which in turn impacts on the calculated $R_{0}$. In reality the two chromophores are unlikely to be truly freely rotating with respect to each other when fused to a protein of interest ${ }^{9}$. Therefore, it is difficult to accurately equate FRET efficiency to distance.

The question which thus arises is how the proximity of two FPs influence fluorescence, including FRET. Many fluorescent proteins, especially those that emit 
in the red region, naturally exist as oligomers ${ }^{10}$ or have a tendency to oligomerise ${ }^{11}$. A great deal of protein engineering effort to generate functional monomeric forms but many commonly used FPs have been shown to have a capacity to dimerise ${ }^{11,12}$. Dimerisation can be compounded by local high concentrations brought about by interactions between the fusion partner proteins that is to be monitored. Thus, when investigating FRET between FPs there may not just be simple spatial proximity at work but molecular interactions leading to more defined distance and dipole alignment, which may in turn influence inherent fluorescence. It has previously been thought that by using FPs from different organism classes with low sequence identities (e.g. GFP with RFPs) should prevent dimerisation.

We ${ }^{13}$ and others ${ }^{14-17}$ have previously shown that FP association can be promoted through either connecting FPs with linker sequences/protein domains, or by forming oligomers from individual monomers. In relation to the current work, we have shown that FP dimers can be constructed via genetically encoded strainpromoted azide-alkyne cycloaddition (SPAAC) ${ }^{13}$, with dimerisation resulting in changes to the spectral properties. Here, we describe the construction and analysis of various Click linked FP dimers (Figure 1a). The structure of an artificial dimer of super-folder GFP (sfGFP) provides a rationale for enhanced fluorescence and role of water dynamics in this process. Using this new structural information, we determined $\kappa^{2}$ values and measured $J(\lambda)$ to calculate more realistic $R_{0}$ values for experimentally analysed Click linked sfGFP-Venus dimers. We find that theoretical FRET efficiency does not match the observed FRET efficiency suggesting that proximity and dipole arrangement may not be the only factors that influence energy transfer. Furthermore, we linked sfGFP and mCherry together and found little FRET between the two proteins, with mCherry fluorescence being largely lost on dimerisation. 

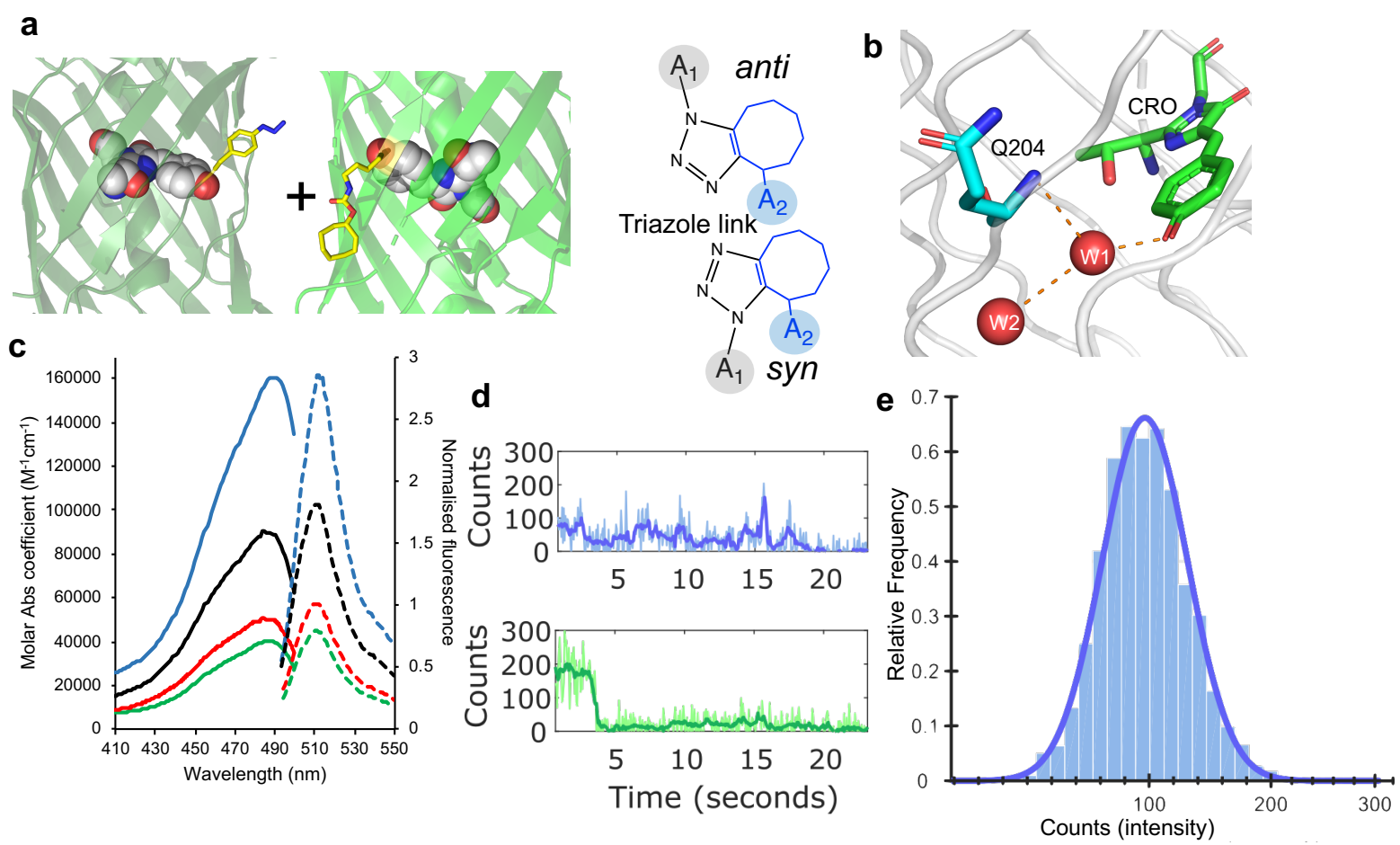

Figure 1. Click-based protein dimerisation via residue 204. (a) Covalent crosslink via genetically encoded $p$-azido-L-phenylalanine (azF) in one monomer and strained-cyclooctyne pyrrolysine (SCO-K) placed in the second monomer. Shown are the two different final regioisomers available. (b) Relative positioning of residue Q204 with respect to chromophore (CRO) and local water molecules (red spheres W1 and W2). (c) Steady state bulk absorbance (full line) and fluorescence emission (dashed line) of sfGFP ${ }^{204 \times 2}$ (blue), sfGFP 204azF (green), sfGFP $2045 c 0$ (red) and the addition of the two monomer spectra (black). The data has been reported previously ${ }^{13}$ and shown here for context. (d) Representative single molecule traces for sfGFP $^{204 \times 2}$ (blue) and sfGFPWT (green) measured by TIRF microscopy. Further example of single molecule traces can be found in Supporting Figure S1 for sfGFP ${ }^{204 \times 2}$ and work by Worthy et al ${ }^{13}$. (e) A single molecule fluorescence intensity histogram for sfGFP $204 \times 2$ consisting of 179 trajectories (2602 spots). The histogram data fits to a single log normal distribution centred around 100 counts.

\section{Results and discussion}

\section{The effect of sfGFP proximity on function}

We have previously reported the construction of artificial FP dimers by Click chemistry through the covalent coupling of genetically encoded of ring-strained cyclooctyne derivative of the pyrrolysine (SCO-K) and p-azido-L-phenylalanine (azF) ${ }^{13}$ (Figure 1a). It should be noted that we do not attempt to change residues at the 
FP dimer interface nor link them in a tandem arrangement using a spacer sequence as has been done in other approaches ${ }^{14-18}$ but model potential naturally occurring interface sites, which are in turn stabilised through a SPAAC link. Regions that do not naturally associate do not promote covalent crosslinking via SPAAC ${ }^{13}$. Thus, our approach stabilises naturally feasible protein interactions.

Residue Q204 in sfGFP lies close to the chromophore (CRO; Figure 1b), with the backbone amine group making an indirect $\mathrm{H}$-bond with $\mathrm{CRO}$ via a conserved structured water molecule, W1. In silico molecular docking revealed that Q204 consistently resided at possible dimer interfaces and is close to a region known to be involved in FP dimerisation ${ }^{12}$. The SCO-K (sfGFP ${ }^{204 S C O}$ ) and azF (sfGFP204azF) containing monomers were subsequently proved to dimerise, generating the dimer termed sfGFP204×2 13. The sfGFP 204-linked dimer displayed enhanced fluorescence compared to the monomers. Dimeric sfGFP204×2 displayed positive functional synergy in which the brightness of the complex was more than the sum of the individual monomers (Figure 1c) ${ }^{13}$. Indeed, sfGFP $204 \times 2$ is brighter on a per CRO basis $\left(57000 \mathrm{M}^{-1} \mathrm{~cm}^{-1}\right)$ than the original sfGFP $\left(37000 \mathrm{M}^{-1} \mathrm{~cm}^{-1}\right){ }^{19,20}$ and EGFP $\left(33000 \mathrm{M}^{-1} \mathrm{~cm}^{-1}\right)^{21}$, two benchmark fluorescent proteins.

The increase in molar absorbance coefficient suggests that the fluorescence lifetime is shorter for sfGFP $204 \times 2(0.92 n s)$ compared to the original monomeric sfGFP (3.2 ns, calculated using the website huygens.science.uva.nl/Strickler_Berg/). Real time single molecule fluorescence of SfGFP $204 \times 2$ was undertaken to explore the mechanism of enhanced capacity of the dimer to absorb and emit light. The dimer has an increased ON time compared to the sfGFPWT (average 0.87s GFP204x2 compared to $0.65 \mathrm{~s}$ for GFPWT ). Analysis of individual traces shows this clearly as sfGFP204×2, takes longer to photobleach compared to sfGFPWT (Figure 1d with additional traces in Supporting Figure 1; also see Worthy et al for WT sfGFP single molecule analysis $\left.{ }^{13}\right)$. The increased $\mathrm{ON}$ times and photobleaching lifetime coupled with the shorter fluorescence lifetime is likely account for the increased fluorescence observed in steady state ensemble measurements. It is notable that the single molecule fluorescence time course traces are more complex, and dynamic compared to sfGFPWT with a range of fluorescent states observed, which could indicate cooperative interaction between the individual monomer units.

Ensemble histograms reveal a single dominant intensity peak is observed at a value equivalent to monomeric sfGFPWT (Figure 1e). This differs from a previously 
described artificial dimer linked via residue 148 (termed sfGFP $\left.{ }^{148 \times 2}\right)^{13}$, which exhibits two distinct population states. If the two molecules in the dimer are acting largely independently of each other, a bimodal distribution would be expected. Thus, only 1 $\mathrm{CRO}$ in the dimer is fluorescent at any given time.

a
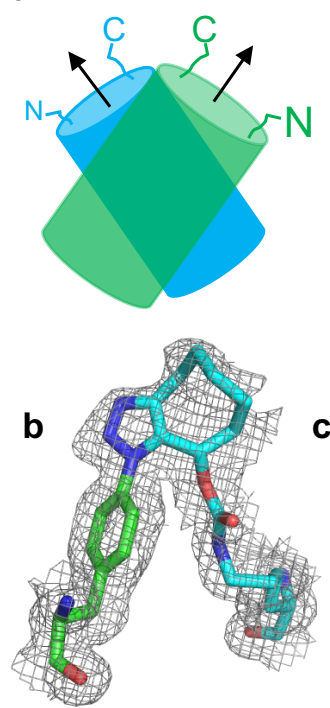
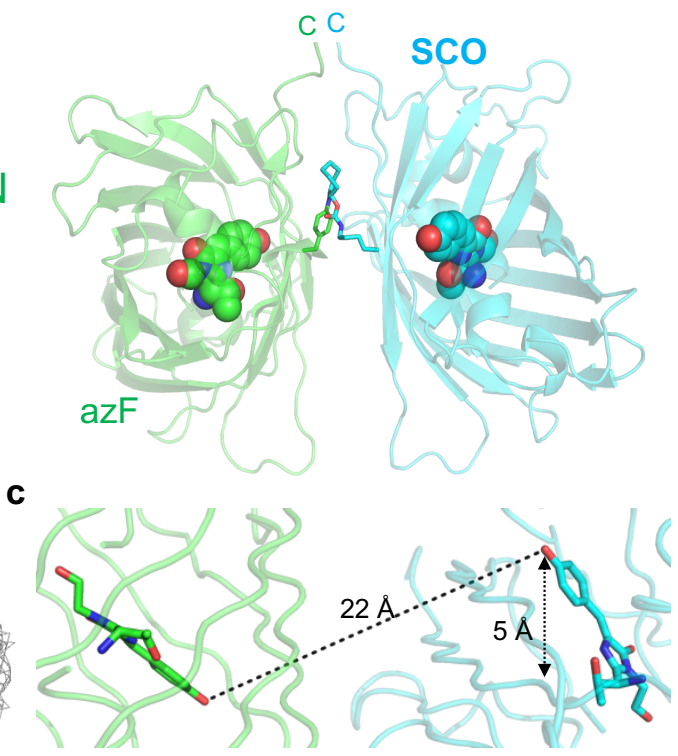

Figure 2. Structure of sfGFP ${ }^{204 \times 2}$. (a) Arrangement of the azF (green) and SCO (blue) containing monomers. (b) The syn conformation of the triazole linkage with the electron density map (2Fo-Fc, 1.0 sigma) shown. (c) Distances and offset of the two CROs (shown as sticks)

\section{Structural basis for proximity-based effects.}

The structure of sfGFP ${ }^{204 \times 2}$ (structural statics in Supporting Table S1 and Supporting Figures S2a-b) reveals that each monomer unit is similar to the original starting sfGFP. The sfGFP204×2 dimer forms a quasi-symmetrical off-set "side-byside" monomer arrangement (Figure 2a), which is promoted by formation of a syn 1,5 triazole link that generates a reverse turn structure (Figure $2 b$ ). The two CROs points towards each other in an antiparallel arrangement $22 \AA$ apart with a $5 \AA$ offset (Figure 2c). It is closest to the 3rd ranked model predicted previously ${ }^{13}$ (Supporting Figure S2b-c). Each monomer is offset by $\sim 70^{\circ}$ with the C-termini close in space (Figure 2b). As the $\mathrm{N}$ - and C-termini are close to each other at the same end of the $\beta$-barrel, the proximity and orientation of the two termini in the dimer may well promote such an interaction in a fusion protein construct. 


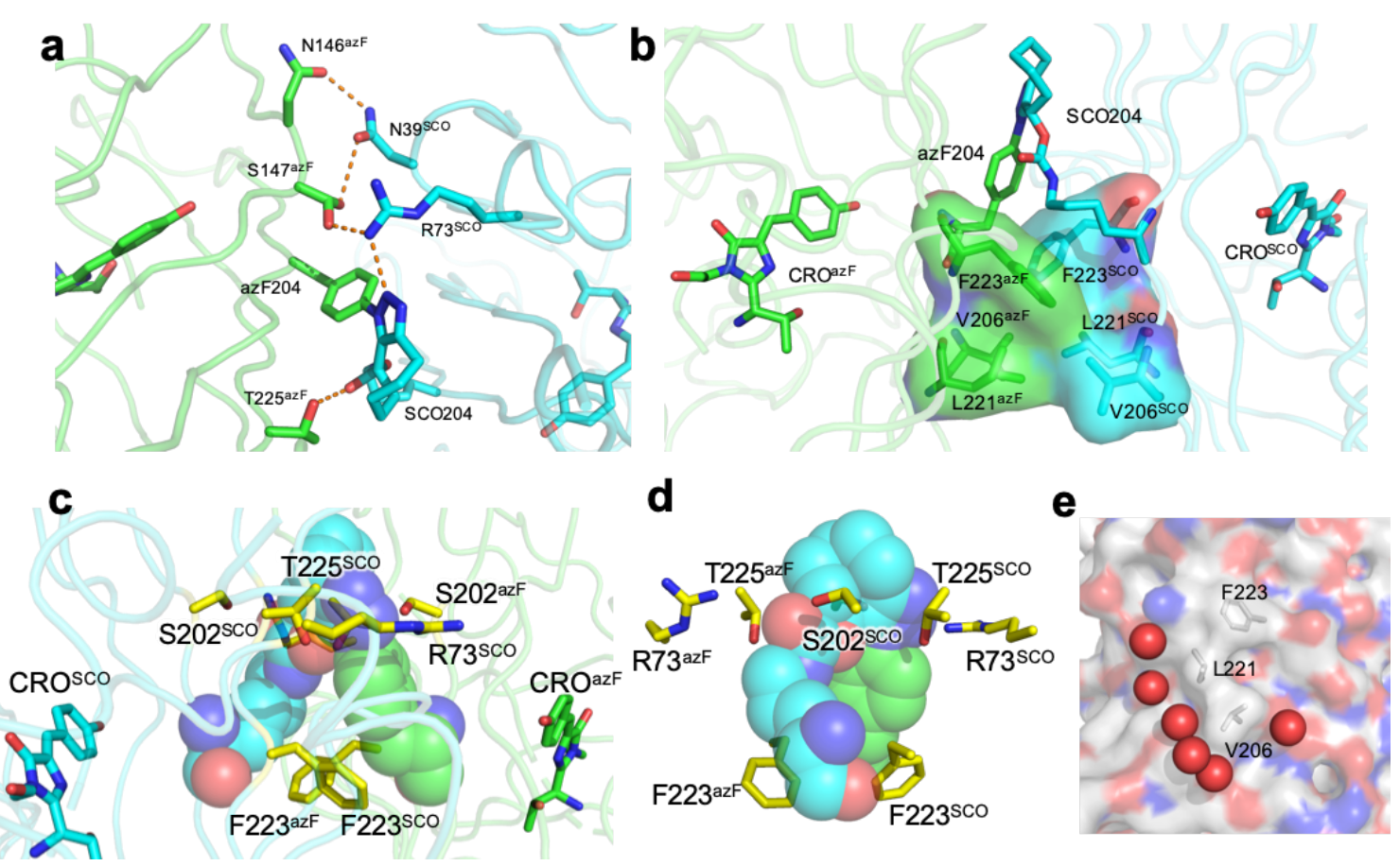

Figure 3. Subunit interface in sfGFP ${ }^{204 \times 2}$ comprised of sfGFP204azF (green) and sfGFP $^{204 S C O}$ (cyan). (a) H-bond network at interface. (b) Hydrophobic interactions. (c and d) Interactions around the triazole link shown in two different orientations. (e) Water molecules (red spheres) associated with the interface region.

The two monomer units associate to form an extensive and intimate interface. While the interface area is relatively small $\left(\sim 900 \AA^{2}\right)$, the main elements that comprise a protein-protein interface, namely hydrophobic interactions and $\mathrm{H}$-bonding are observed (Figure 3). The H-bond network at the interface is not symmetrical but the hydrophobic interactions show a significant degree of symmetry (Figure 3a). The hydrophobic core interface is comprised of Phe223, Val206, Leu221 from both chains interlocking (Figure $3 b$ ). These residues are surface exposed in sfGFP and form a naturally occurring hydrophobic patch ${ }^{12}$ that can facilitate and stabilise the dimer on Click crosslinking (Figure 3e), or for that matter potentially other FPs. Indeed, mutation of Val206 to a charged residue is known to reduce dimerisation tendency of $A$. victoria derived GFPs ${ }^{12}$.

The new triazole crosslink is integrated within the structure being semi-buried at the dimer interface and lying above the plane of the main hydrophobic interface patch (Figure 3c-d). The azF component is fully buried while one face of the SCO moiety is partially accessible to the solvent. Phe223 from both monomers forms the base of the triazole reverse turn (Figure 3c-d) while Arg73, Ser202 and Thr225 
residues make putative polar interactions with oxygen and nitrogen atoms in the SCO-azide link. A more extended network linking the two chromophores is proposed in Supporting Figure S3. Thus, the new crosslink is not just a simple chemical bolt link between the two monomers but forms an integral structural component.
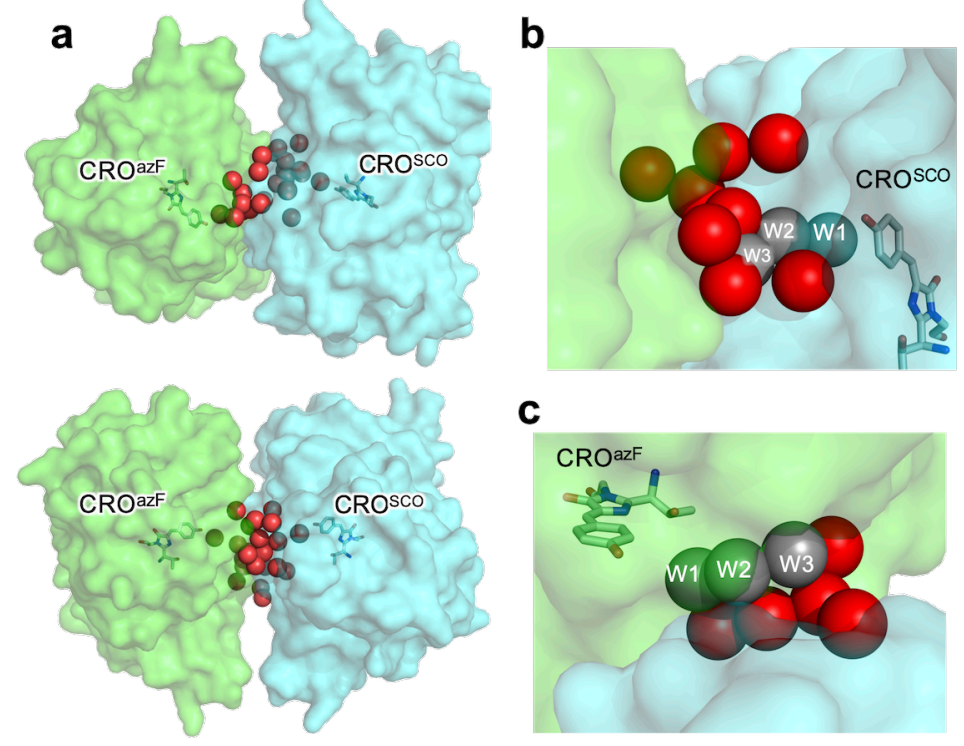

Figure 4. Water-rich cavities at dimer interface. (a) water (red spheres) filled cavities shown from two different angles. Waters associated with the (b) sfGFP ${ }^{204 s c o} \mathrm{CRO}$ and (c) sfGFP204azF CRO. The grey spheres are equivalent to W1, W2 and W3 shown in Figure 1 and Supporting Figure S4. Waters molecules W2 and W3 are observed in the sfGFPWT structure but are largely surface exposed (Figure S4).

At the interface are two cavities filled with ordered water molecules (Figure 4a). The water molecules are arranged around an area where the chromophore protrudes towards the surface. A partially buried water molecule (W1; Figure 1c and Supporting Figure S4) is commonly observed associated with the chromophore via a $\mathrm{H}$-bond with the hydroxyl group and the backbone of residue 204; this water is associated with 1 to 2 additional surface water molecules (grey spheres, Figure 4b-c) as observed for monomeric sfGFPWT (Supporting Figure S4). In the sfGFP204x2 dimer, these waters lie within the cavity together with several additional tightly packed water molecules. The roles of the additional waters associated with W1 in terms of their impact on the structure-function relationship is not fully known but it has been postulated that they contribute to charge transfer and modulating the protonated state of the CRO ${ }^{22-24}$. In solution, it is likely that the additional water molecules associated with $\mathrm{W} 1$ are in free exchange with the solvent when sfGFP is 
monomeric; exchange with bulk solvent is likely to be minimal in the dimeric sfGFP $204 \times 2$ so persist in a defined arrangement for longer. By changing the dynamics of normally surface associated water molecule could potentially contribute towards the enhanced brightness observed on dimerisation through the formation of more persistent networks.

a

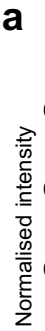

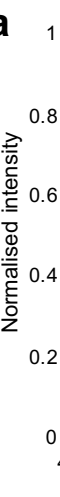

b

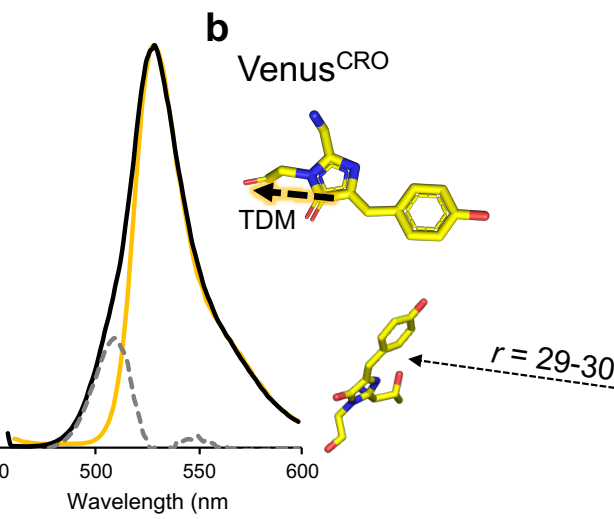

SfGFPCRO

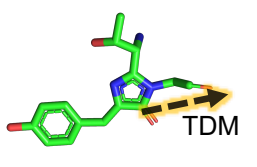

C
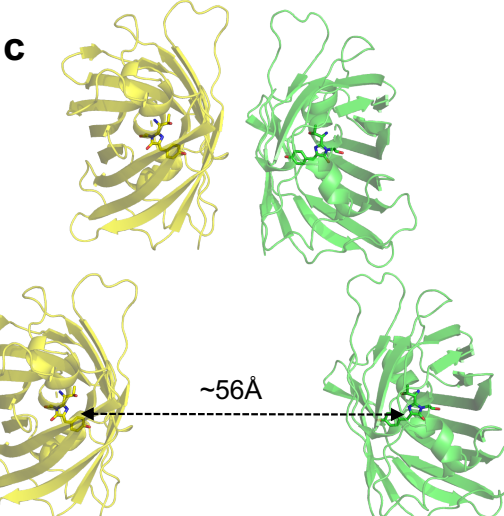

Figure 5. Energy transfer between sfGFP and Venus. (a) Fluorescence emission spectra of GFVen $^{204}$ (black), Venus ${ }^{204 a z F}$ (gold) and the residual emission profile highlighting sfGFP contribution to GFVen ${ }^{204}$ spectrum (grey dashed). Excitation was at $450 \mathrm{~nm}$. (b) Relative positionings of the Venus (yellow) and sfGFP (green) chromophores (CROs) in the model GFVen 204 structure. The dashed lines highlighted in orange represent the transition dipole moment (TDM). (c) Relative distances between Venus (yellow) and sfGFP (green) based on the model of SPAAC linked dimer (top) and observed FRET efficiency (bottom). Structures are to scale.

\section{Heterodimers and functional communication by energy transfer}

The use of different FPs with compatible spectral properties to promote FRET is essential for biomolecular analysis. The sfGFP $2045 \mathrm{SO}$ variant can be linked to Venus (containing azF) via residue 204 to generate heterodimers ${ }^{13}$. The resulting dimer, termed GFVen ${ }^{204}$, demonstrated FRET from the sfGFP component to Venus, as would be expected (Figure 5a). There is currently very little known about the relative orientation of FRET-based FP pairs with only one structure available in a biosensor configuration ${ }^{14}$, which is in a single polypeptide format rather that a classical twoprotein system. Given the high degree of sequence and structure similarity between sfGFP and Venus, we used the GFP $204 \times 2$ structure to build models of the GFVen 204 dimer so as to calculate more specific $R_{0}$ factors based on the relative orientations of 
the two chromophores (Supporting Figure 5). Using our model of GFVen ${ }^{204 \times 2}$ together with the known transitions dipole arrangements for both GFP and Venus 25,26 (Figure 5b), $\kappa^{2}$ was calculated in the model to be 3.59. Using the $Q_{D}$ and $J(\lambda)$ values (Supporting Table S2) together with a refractive index of 1.4 to account for a combined protein-water environment (Hellenkamp et al ${ }^{9}$ and Dr Tim Craggs personal communication via Twitter) we calculated $R_{0}$ with the different $\kappa^{2}$ values (Supporting Table S2). The calculated $\mathrm{R}_{0}$ differs were $\sim 76 \AA$, which is up to $19 \AA$ longer when calculated using the arbitrary $0.667 \kappa^{2}$ value. Our calculated $R_{0}$ values are consistent with those calculated using $J(\lambda)$ and donor QY values available through FPbase (https://www.fpbase.org) ${ }^{27}$ when adjusted for $\kappa^{2}$ (see Supporting Table S2).

The question arises is how does our calculated $R_{0}$ relate through to observed FRET efficiency. Based on the use of equation 2 and the measured interchromophore distance of 29-30 $\AA$ (Figure 5b), the estimated FRET efficiency for our GFVen ${ }^{204}$ construct should be close to $100 \%$ (99.6-99.7\%). However, deconvolution of the GFVen ${ }^{204}$ emission on excitation at $450 \mathrm{~nm}$ (a wavelength that will excite only sfGFP) reveals a significant sfGFP component (Figure 5a). Using equation 3, FRET efficiency can be calculated.

$$
E_{\text {rel }}=I_{\mathrm{A}} /\left(I_{\mathrm{D}}+I_{\mathrm{A}}\right) \quad \text { equation } 3
$$

where $E_{\text {rel }}$ is relative FRET efficiency, $I_{\mathrm{A}}$ is integrated fluorescence of the acceptor and $I_{D}$ is the integrated fluorescence of the donor. FRET efficiency was determined to be $86.8 \%$. Thus, there is a clear discrepancy between the observed and theoretical FRET efficiency, which has been observed before form structure-based analysis where inter-FP interactions were observed ${ }^{7,14}$. What gives rise to this difference? A simple and obvious explanation is that some free monomeric sfGFP $2045 C O$ is present. Analysis of polyacrylamide gels and mass spectrum suggests little or no monomeric protein is present (see Worthy et al ${ }^{13}$ and Supporting Figure S6 for details). Are the considerable number of water molecules present at the domain interface observed for sfGFP $204 \times 2$ (Figure 4) playing a role in quenching? Water can quench fluorescence ${ }^{7,28}$, especially if collisional events are promoted through free dynamic exchange. However, the crystal structure suggests local water molecules are likely to be less dynamic in the dimer compared to monomeric forms. Is the arrangement of the monomers in GFVen ${ }^{204 \times 2}$ similar to the 
assumed sfGFP204×2? While we cannot rule out some rotation of one FP with respect to another, the triazole link will restrict such rotation and the CROs will retain a similar vector configuration in terms of the transition dipole moments. With a $R_{0}$ of $76.62 \AA$, the two CROs will need to be at least $50 \AA$ apart (shown schematically in Figure $5 c$ ). Even using the arbitrary $\kappa^{2}$ value of 0.667 generates a $R_{0}$ of $56 \AA$, which will require the CROs to be $\sim 40 \AA$ apart to generate the observed FRET efficiency. Given the relationship of residue 204 to the CRO (Figure 1), neither distances are feasible in a covalently linked dimer. It is clear that bringing two different FPs in close proximity so promoting inter-FP interactions can influence FRET efficiency, which results in an overestimation of the distance between the pair.
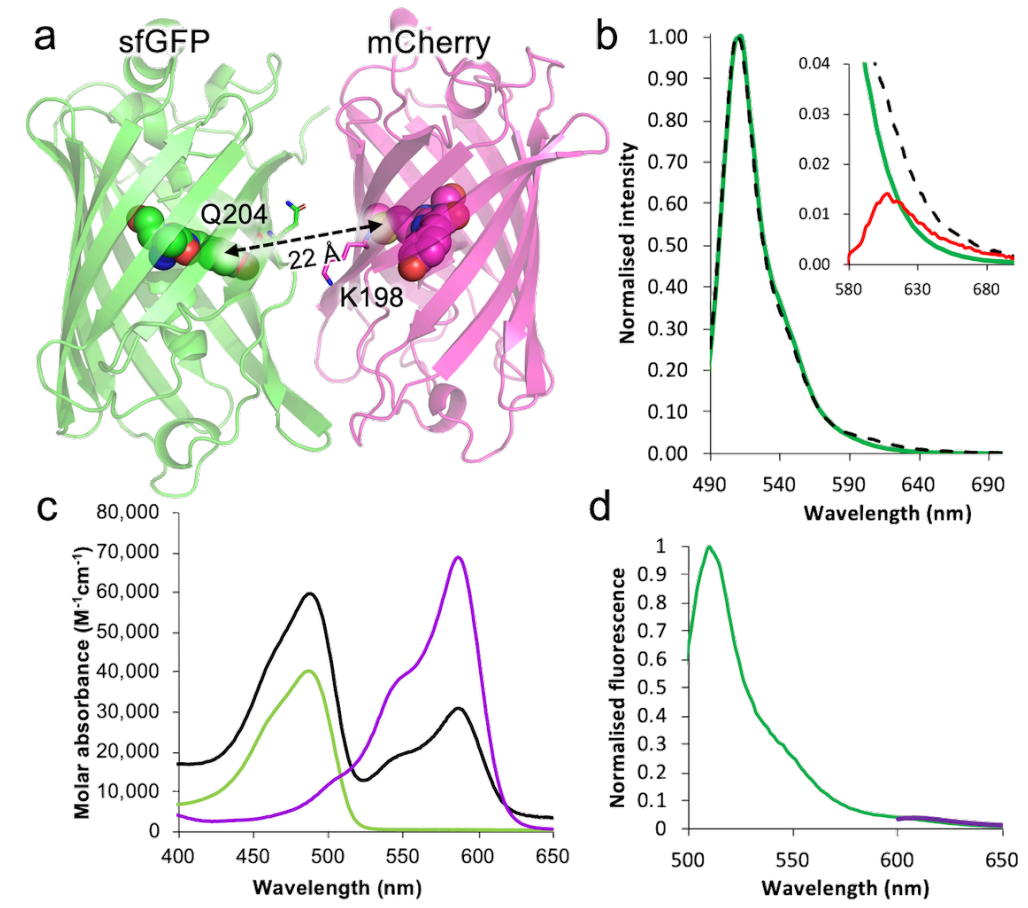

Figure 6. Effect on function of proximally located sfGFP and mCherry. (a) Modelled docking of mCherry (PDB: 2h5q) and sfGFP (PDB: 2b3p) using ClusPro ${ }^{29}$. Residues Q204 (sfGFP) and K198 (mCherry) that are replaced by SCO-K and azF, respectively, are highlighted. (b) Fluorescence emission (on excitation at $485 \mathrm{~nm}$ ) of sfGFP ${ }^{204 S C O}$ (green solid line) and $\mathrm{GFCh}^{\times 2}$ dimer (dashed black line). Emission intensities are normalised to sfGFP204Sco. Inset is the zoom in region of the emission spectrum centred around $610 \mathrm{~nm}$, the emission maximum of mCherry. The red corresponds to the subtraction of $\mathrm{GFCh}^{\times 2}$ from sfGFP ${ }^{204 \mathrm{SCO}}$. (c) Molar absorbance of $\operatorname{sfGFP}^{204 S C O}$ (green), mCherry ${ }^{198 a z F}$ (purple) and GFCh ${ }^{\times 2}$ (black line). (d) Emission profile of $\mathrm{GFCh}^{\times 2}$ on excitation at $485 \mathrm{~nm}$ (green) and $585 \mathrm{~nm}$ (purple). 


\section{Proximity effect of green and red fluorescent proteins.}

We next linked together sfGFP with a DsRed derived monomeric protein, mCherry 30,31. Green fluorescent proteins can be used as a FRET partner with mCherry ${ }^{16,32-34}$ with an estimated $J$ coupling of $1.8 \times 10^{15} \mathrm{M}^{-1} \mathrm{~cm}^{-1} \mathrm{~nm}^{4}$ (FPbase FRET tool (www.fpbase.org/fret/) ${ }^{27}$. The sfGFP 204 ScO variants was reacted with mCherry containing azF at the structurally equivalent position, residue 198 (Figure 6a). Molecular docking suggested the two proteins can associate at the interface between residues $204^{\text {sfGFP }}$ and $198^{\text {mCherry }}$ (Figure 6a), with covalent coupling via SPAAC subsequently proved by SDS PAGE (Supporting Figure S7). Incorporation of azF at residue 198 in mCherry had little effect on the spectral properties of the momomer with a similar molar absorbance and brightness to the wt mCherry $(69,000$ $\mathrm{M}^{-1} \mathrm{~cm}^{-1}$ with a quantum yield of $24 \%$ compared to $72,000 \mathrm{M}^{-1} \mathrm{~cm}^{-1}$ for wt mCherry with quantum yield of $22 \%$ at $587 \mathrm{~nm}$; Figure $6 \mathrm{c}$ and Supporting Figure S8a).

The purified dimer, termed GFCh ${ }^{\times 2}$ did not appear to display any significant FRET on excitation at $490 \mathrm{~nm}$ (Figure 6b). Indeed, very little observable fluorescence can be attributed to mCherry in the dimer even on excitation at $585 \mathrm{~nm}$ (Figure 6d), which is confirmed visually through general UV excitation (Supporting Figure S7b). The mCherry associated peak at $\sim 585 \mathrm{~nm}$ is reduced in terms of molar absorbance compared to the mCherry ${ }^{198 a z F}$ monomer. As with other dimeric forms (vide supra and ${ }^{13}$ ), the sfGFP molar absorbance increased above the simple addition of the two monomeric forms $\left(\sim 16,000 \mathrm{M}^{-1} \mathrm{~cm}^{-1}\right.$ taking into account the contribution from the mCherry chromophore) confirming the role of dimerisation via residue 204 in enhancing sfGFP function. Data suggests that the interaction between the SfGFP and $\mathrm{mCherry}$ is responsible for loss of fluorescence. Reaction with the SCO-K ncAA alone does not appear to affect fluorescence (Supporting Figure S8b). Attachment with the bulker azide containing Cy3 dye also does not result in loss of fluorescence, with FRET observed as expected (Supporting Figure S8c). Thus, placing FPs in close proximity to promote their interaction is the likely course of the loss in fluorescence, in this case from mCherry. As donor fluorescence is still observed, in a FRET experiment this could be interpreted as two proteins not interacting when the opposite may in fact be the case. 

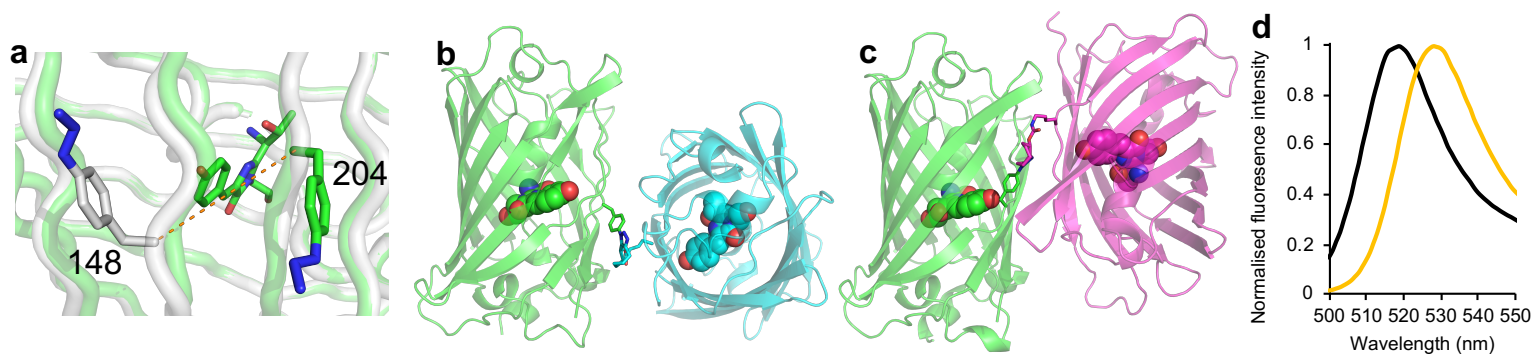

Figure 7. Comparison of sfGFP $204 \times 2$ structure with sfGFP ${ }^{148 \times 2}$. (a) Location of the azF moiety at residue 148 (grey) or 204 (green). Structure of (b) sfGFP $204 \times 2$ and (c) sfGFP ${ }^{148 \times 2}$. Each structure is orientated identically with respect to the azF containing ncAA (coloured green) to highlight the relative differences in monomer arrangements. (d) Normalised emission of on excitation at $490 \mathrm{~nm}$ for GFVen ${ }^{148}$ (black) and wt Venus (gold).

\section{Comparison with alternative sfGFP dimer sfGFP ${ }^{148 \times 2}$}

The structure of another Click-linked artificial dimer joined via residue 148 (termed sfGFP ${ }^{148 \times 2}$ ) has recently been reported ${ }^{13}$. Dimerisation effectively switched sfGFP ${ }^{148 \times 2}$ on, with the dimer displaying improved function compared to both monomers and the original wild type sfGFP (sfGFPWT). We used the structure of the sfGFP ${ }^{148 \times 2}$ dimer to calculate $\kappa^{2}$ as a representative alternative CRO arrangement. This will in turn allow us to investigate how different configurations of one monomer to the other affect dipole alignments and hence FRET. Residue 204 lies close to 148 on the adjacent $\beta$-strand (Figure 7a) but they adopt very different sidechain and thus monomer arrangements in their crystal structures (Figure $7 b-c)$. In contrast to sfGFP $^{204 \times 2}$, the triazole link in sfGFP ${ }^{148 \times 2}$ forms the extended anti form that is reenforced with both polar and hydrophobic interactions between the monomers generating a quasi-symmetrical "head-to-tail" arrangement the monomers. The result of such a configurational change between the two monomers units results in the relative positioning of the $\mathrm{CROs}$ being very different (compare Figure $7 \mathrm{~b}$ and $7 \mathrm{c}$ ).

Using the same approach as for GFVen ${ }^{204}$, we calculated $\kappa^{2}$ values for a model of GFVen ${ }^{148}$ based on the sfGFP ${ }^{148 \times 2}$ structure. It should be noted that unlike linkage through residues 204 (or 198 in mCherry), covalent coupling via residue 148 was designed to instigate a functional change through synergistic conformation events ${ }^{13}$. However, it does allow us to assess how changing the orientation and inter-unit interactions of one monomer to another along a quasi-similar interface regions alters dipole arrangements. The calculated $\kappa^{2}$ was 3.79 , even closer to the maximal value 
of 4 than sfGFP $204 \times 2$. While this would suggest an even longer $\mathrm{R}_{0}$ distance than GFVen ${ }^{204}$, the inherent function of the GFVen ${ }^{148}$ dimer system makes calculating $R_{0}$ problematic; the donor, sfGFPsc0148, is essentially switched off in monomeric state and only becomes activated on dimerisation. However, the main effect that will influence any FRET analysis is the shift in $\lambda_{\mathrm{EM}}$, which is blue shifted by $10 \mathrm{~nm}$ in the GFVen $^{148}$ dimer compared to Venus ${ }^{W T}$ (Figure $7 d$ ) when excited at a wavelength corresponding to sfGFP. If single wavelength readings are taken with $530 \mathrm{~nm}$ assumed to be the Venus emission maximum, fluorescence emission would be underestimated by up to $35 \%$ so impacting on perceived FRET efficiency. While residue 204 is more applicable in terms of understanding proximity and FRET due to the non-perturbative nature of the initial mutations, sfGFP ${ }^{148 \times 2}$ and GFVen ${ }^{148}$ still acts as good examples of how proximity is once again having a significant effect on the spectral properties. It also demonstrates that FP dimerisation are not restricted to a defined interaction configuration but that different inter-FP orientations are available.
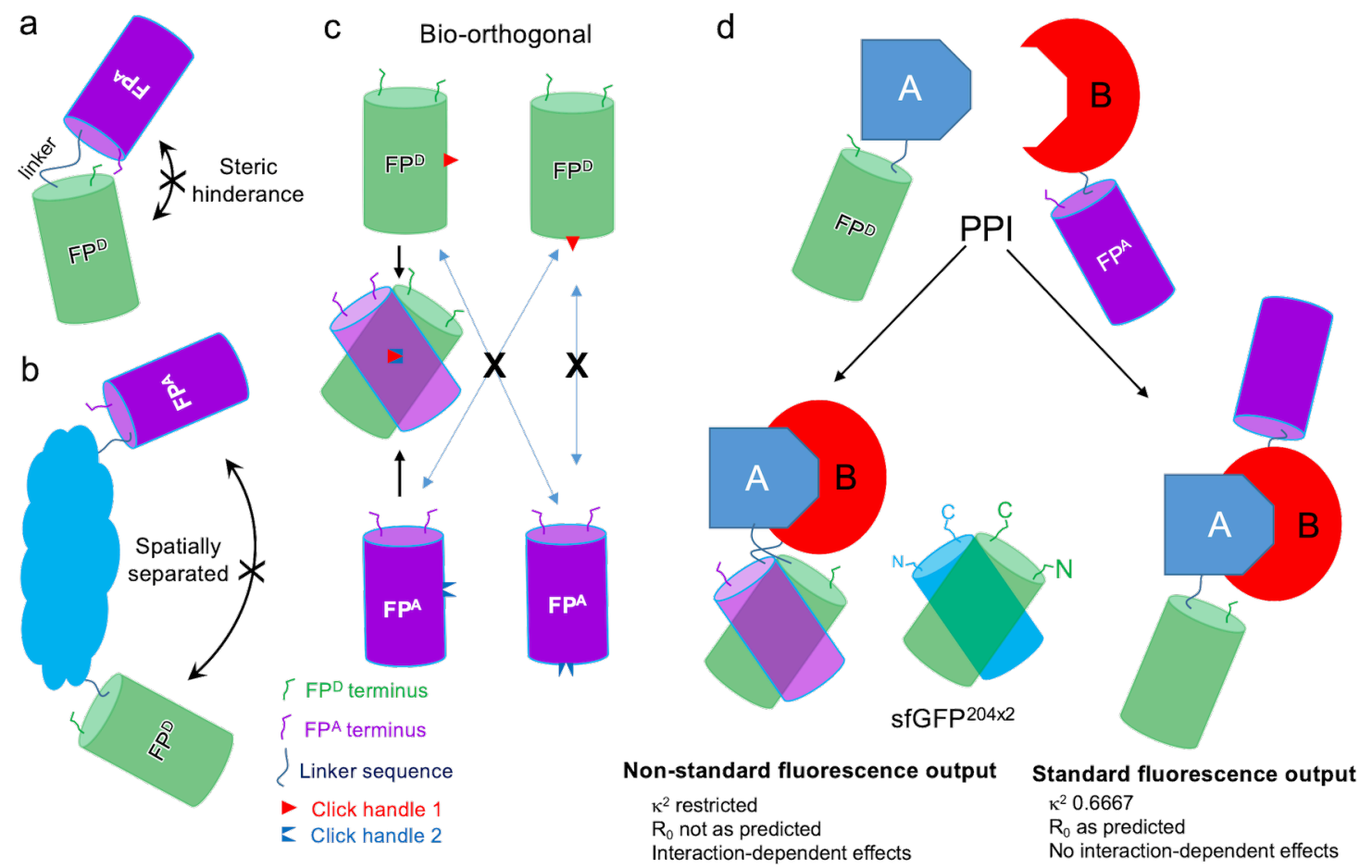

Figure 8. Schematic outline of assessing FP pairs. FPD and $\mathrm{FP}^{\mathrm{A}}$ refer to nominal FP donor and acceptor for FRET. (a) Model system where short linkers generating a tandem FP pair. Linkers may be too short to allow full freedom to sample interactions, especially side-on interactions. So, there is a low likelihood of FP interaction. (b) Model system whereby FP pairs are bridged by a protein domain or whole protein. If the termini of the bridging protein are at opposite ends then the two FPs will be spatially separated so cannot interact. (c) Bio-orthogonal Click chemistry approach whereby one FP has one type of chemistry (e.g. azide) and the second a mutually reactive handle (shown as red triangle and blue inverted triangle). Only FPs with mutually compatible interfaces will react and so stabilise the interaction. If the interface is not compatible the FPs will not click together. Broader FP-FP interface regions can be sampled through this 
approach. (d) Protein-protein interaction (PPI) system. Two scenarios are envisaged. The first is that on interaction of protein A and B, the FPs are brought in close proximity to each other promoting association, which may in turn lead to non-standard fluorescence properties. In the second scenario, protein A and B interact but the FPs remain spatially separate so displaying more classical fluorescence behaviour.

\section{Conclusion}

Our ability to construct artificial dimers of FPs coupled with structural analysis has allowed us to look at how proximity can influence two of their key functions: inherent electronic excitation/light emission and communication through energy transfer. With regards to the latter, we can use structural information to predict dipole alignments of two CROs, which is critical to FRET through defining $\kappa^{2}$. In our case, the arbitrary 0.6667 for the $\kappa^{2}$ value provides a significant underestimate of the predicted values that impacts on $R_{0}$. There are been several studies to date that measure FRET in artificial constructs whereby FPs are coupled via linker sequences or whole protein domains. However, by linking two FPs together they can no longer freely interact with each other due to, for example, steric hinderance (e.g. when using linker sequences) ${ }^{15}$ or spatially forced apart (e.g. when linked to whole proteins) ${ }^{14}$, schematically outlined in Figure 8. Our use of bio-orthogonal chemistry allows broader sampling and stabilisation of mutually compatible FP interfaces (Figure 8c); non-compatible FP surfaces do not form covalent bonds so the interface will not persist ${ }^{13}$. The most powerful use of FRET is monitoring protein-protein interactions whereby the FPs are fused to separate proteins. It can be argued that most FP fusions will not associate in most FRET experiments. However, as FPs will be attached to partner protein that normally associate, and if the two FPs are in close proximity they may well align or even interact in preferential arrangements (Figure 8d). This in turn can affect dipole alignment and even inherent FP function. Naïve docking of FPs along with empirical evidences highlights FPs tendency to oligomerise, which will be enhanced by local high concentrations. It is thus clear from our work that by placing FPs in close proximity can result in changes in the expected fluorescence behaviour.

\section{Methods.}


Protein production. The monomeric sfGFP $204 a z F$ and sfGFP 204 SCO proteins and the sfGFP ${ }^{204 \times 2}$ dimer were produced as described previously ${ }^{13}$. The WT mCherry and $\mathrm{mCherry}{ }^{198 a z F}$ proteins were produced as outlined in the Supporting Methods.

Protein dimerisation and conjugation. The procedures for generating sfGFP homodimers and sfGFP-Venus heterodimers have been reported previously ${ }^{13}$. Generation of the sfGFP-mCherry dimers was performed as follows. The sfGFP $^{204 S C O}$ and mCherry ${ }^{198 a z F}$ were mixed at a equimolar concentration $(50 \mu \mathrm{M}, 50$ $\mathrm{mM}$ Tris- $\mathrm{HCl} \mathrm{pH} \mathrm{8.0)} \mathrm{and} \mathrm{left} \mathrm{at} \mathrm{room} \mathrm{temperature} \mathrm{for} \sim 16 \mathrm{hr}$. Dimers were purified by size exclusion chromatography (Superdex 200,16/600) and protein concentration determined, as described above. Protein dimerisation and separation was also monitored by SDS PAGE gel. Conjugation with non-proteinaceous molecules is described in the Supporting Methods.

Steady state absorbance and fluorescence analysis. Spectrophotometry and fluorescence were performed essentially as described previously for sfGFP monomers and dimers, Venus monomers and sfGFP-Venus hybrid dimers ${ }^{13}$. Analysis of variants involving mCherry followed a similar analysis procedure, using proteins concentration of $5 \mu \mathrm{M}$ in $50 \mathrm{mM}$ Tris- $\mathrm{HCl} \mathrm{pH} \mathrm{8.0.} \mathrm{Absorbance} \mathrm{spectra} \mathrm{were}$ recorded on a Cary Win UV, using a $300 \mathrm{~nm} / \mathrm{min}$ scan rate at $1 \mathrm{~nm}$ intervals. Absorbance at $\lambda_{\max }$ for each variant, was used to determine the molar extinction coefficients $(\varepsilon)$ for each variant, using the Beer-Lambert equation and measured protein concentrations. Emission spectra were collected on a Cary Varian fluorimeter at a scan rate of $60 \mathrm{~nm} / \mathrm{min}$ and $1 \mathrm{~nm}$ intervals. Emission and excitation slit widths were set to $10 \mathrm{~nm}$ and a detector voltage of Low. Samples were excited at $5 \mathrm{~nm}$ from $460 \mathrm{~nm}$ to $590 \mathrm{~nm}$ as stated in the main text and emission was scanned from the excitation wavelength to $800 \mathrm{~nm}$. J coupling constants $(J(\lambda))$ were calculated using either available parameters on FPbase ${ }^{27}$ via the FRET tool or calculated from experimental data using ale software (http://www.fluortools.com/software/ae). FRET efficiency was calculated using Equation 3.

Single molecule fluorescence. Measurement and analysis of single molecule sfGFP $204 \times 2$ fluorescence by total internal resonance fluorescence microscopy was performed as described previously ${ }^{13}$. 
Structure determination of sfGFP ${ }^{204 \times 2}$. The sfGFP $204 \times 2$ dimer variant was concentrated in $50 \mathrm{mM}$ Tris $\mathrm{pH} 8.0$ to a final concentration of $10 \mathrm{mg} / \mathrm{ml}$, and used to set up vapour diffusion crystal trays. A JBScreen membrane (Jena Bioscience, Germany) was used initially to facilitate crystal growth, where large green crystals grew in a multitude of buffer conditions. Large green crystals grew in $20 \%$ polyethylene glycol w/v, $100 \mathrm{mM}$ HEPES, which were harvested and transferred to mother liquor supplemented with 13\% (w/v) PEG 200 as a cryo-protectant, and vitrified in liquid nitrogen. X-ray scattering data was collected at the Diamond light source, Harwell, UK (beamline IO2). Structure refinement was performed using the CCP4 program suite ${ }^{35}$. The structure was solved initially using the molecular replacement program PHASER ${ }^{36}$, with wt sfGFP (PDB accession 2B3P) used as a model. Structures were manually adjusted using with $\mathrm{COOT}^{37}$, and refined with TLS restrained refinement using REFMAC ${ }^{38}$.

$\mathrm{Kappa}^{2}$ calculation. The dipole orientation factor, $\mathrm{K}^{2}$, was calculated using an approach as described previously ${ }^{39}$. The model structure of GFVen ${ }^{204}$ dimer was built by overlapping the WT structure of Venus (1MYW) onto the sfGFP204azF component of sfGFP204×2 structure. While Venus and sfGFP used here have 15 amino acid differences in the core $\beta$-barrel structure only Ala206 in sfGFPSCO204 and Val206 in Venus contribute to the domain interface. Residue 204 was then replaced with azF and linked to SCO using the PyMOL bond building tool. The GFVen 204 model overlaid with sfGFP $204 \times 2$ is shown in Supporting Figure S4. Using the model structure the transition dipole moment (TDM) was calculated using the distance between the centres of the donor and acceptor dyes, and the orientations of the transition dipole moments of the donor, and the acceptor ${ }^{39}$. The atomic positions of CG2 and C2 of the chromophore, as shown in Supporting Figure S9, were used to the define the vector for the TDM for both Venus and sfGFP ${ }^{25}$. The $\kappa^{2}$ was then used in equation 1 together with available experimental to calculate $R_{0}$ for FRET pairs.

\section{Acknowledgements}

We would like to thank the staff at the Diamond Light Source (Harwell, UK) for the supply of facilities and beam time, especially Beamline 103 and 104 staff. We would also like to thank Edward Lemke and his group at EMBL Heidelberg for donating the 
pEVOL-SCO plasmid. We thank BBSRC (BB/H003746/1 and BB/M000249/1), EPSRC (EP/J015318/1) and Cardiff SynBio Initiative/SynBioCite for supporting this work. RLJ was supported by a Knowledge Economy Skills Scholarship (KESS2) PhD studentship, WDJ by Wellcome ISSF, HLW. by a BBSRC-facing Cardiff University PhD studentship, HSA. by the Higher Committee for Education Development in Iraq. We would like to thank the Protein Technology Hub, School of Biosciences, Cardiff University for use of facilities. We also thank $\mathrm{Dr}$ Joachim Goedhart from the University of Amsterdam for his helpful and insightful comments on the manuscript and providing the scripts for calculating fluorescence lifetimes form molar absorbance coefficients.

\section{References}

1. Rodriguez, E.A. et al. The Growing and Glowing Toolbox of Fluorescent and Photoactive Proteins. Trends Biochem Sci 42, 111-129 (2017).

2. Duwe, S. \& Dedecker, P. Optimizing the fluorescent protein toolbox and its use. Curr Opin Biotechnol 58, 183-191 (2019).

3. Shaner, N.C., Steinbach, P.A. \& Tsien, R.Y. A guide to choosing fluorescent proteins. Nat Methods 2, 905-9 (2005).

4. Newman, R.H., Fosbrink, M.D. \& Zhang, J. Genetically encodable fluorescent biosensors for tracking signaling dynamics in living cells. Chem Rev 111, 3614-66 (2011).

5. Miyawaki, A., Shcherbakova, D.M. \& Verkhusha, V.V. Red fluorescent proteins: chromophore formation and cellular applications. Curr Opin Struct Biol 22, 679-88 (2012).

6. Ibraheem, A. \& Campbell, R.E. Designs and applications of fluorescent protein-based biosensors. Curr Opin Chem Biol 14, 30-6 (2010).

7. Tsien, R.Y. The green fluorescent protein. Annual review of biochemistry 67, 509-44 (1998).

8. Förster, T. Zwischenmolekulare Energiewanderung und Fluoreszenz. Ann. Phys. 437, 55-75 (1948).

9. Hellenkamp, B. et al. Precision and accuracy of single-molecule FRET measurements-a multi-laboratory benchmark study. Nat Methods 15, 669-676 (2018).

10. Matz, M.V. et al. Fluorescent proteins from nonbioluminescent Anthozoa species. Nat Biotechnol 17, 969-73 (1999).

11. Cranfill, P.J. et al. Quantitative assessment of fluorescent proteins. Nat Methods 13, 557-62 (2016).

12. Zacharias, D.A., Violin, J.D., Newton, A.C. \& Tsien, R.Y. Partitioning of lipidmodified monomeric GFPs into membrane microdomains of live cells. Science 296, 913-6 (2002).

13. Worthy, H.L. et al. Positive functional synergy of structurally integrated artificial protein dimers assembled by Click chemistry. Communications Chemistry 2, 83 (2019). 
14. Trigo-Mourino, P., Thestrup, T., Griesbeck, O., Griesinger, C. \& Becker, S. Dynamic tuning of FRET in a green fluorescent protein biosensor. Sci Adv $\mathbf{5}$, eaaw4988 (2019).

15. Wiens, M.D. et al. A Tandem Green-Red Heterodimeric Fluorescent Protein with High FRET Efficiency. Chembiochem 17, 2361-2367 (2016).

16. Lam, A.J. et al. Improving FRET dynamic range with bright green and red fluorescent proteins. Nat Methods 9, 1005-12 (2012).

17. Lindenburg, L.H., Hessels, A.M., Ebberink, E.H., Arts, R. \& Merkx, M. Robust red FRET sensors using self-associating fluorescent domains. ACS Chem Biol 8, 2133-9 (2013).

18. Mastop, M. et al. Characterization of a spectrally diverse set of fluorescent proteins as FRET acceptors for mTurquoise2. Sci Rep 7, 11999 (2017).

19. Reddington, S.C. et al. Different photochemical events of a genetically encoded phenyl azide define and modulate GFP fluorescence. Angew Chem Int Ed Engl 52, 5974-7 (2013).

20. Reddington, S.C., Tippmann, E.M. \& Jones, D.D. Residue choice defines efficiency and influence of bioorthogonal protein modification via genetically encoded strain promoted Click chemistry. Chem Commun (Camb) 48, 841921 (2012).

21. Arpino, J.A., Rizkallah, P.J. \& Jones, D.D. Crystal structure of enhanced green fluorescent protein to 1.35 a resolution reveals alternative conformations for glu222. PloS one 7, e47132 (2012).

22. Hartley, A.M., Worthy, H.L., Reddington, S.C., Rizkallah, P.J. \& Jones, D.D. Molecular basis for functional switching of GFP by two disparate non-native post-translational modifications of a phenyl azide reaction handle. Chemical Science (2016).

23. Shinobu, A. \& Agmon, N. Proton Wire Dynamics in the Green Fluorescent Protein. J Chem Theory Comput 13, 353-369 (2017).

24. Shinobu, A., Palm, G.J., Schierbeek, A.J. \& Agmon, N. Visualizing proton antenna in a high-resolution green fluorescent protein structure. J Am Chem Soc 132, 11093-102 (2010).

25. Ansbacher, T. et al. Calculation of transition dipole moment in fluorescent proteins--towards efficient energy transfer. Phys Chem Chem Phys 14, 410917 (2012).

26. Kyrychenko, A., Rodnin, M.V., Ghatak, C. \& Ladokhin, A.S. Joint refinement of FRET measurements using spectroscopic and computational tools. Anal Biochem 522, 1-9 (2017).

27. Lambert, T.J. FPbase: a community-editable fluorescent protein database. Nat Methods 16, 277-278 (2019).

28. Andrews, B.T., Schoenfish, A.R., Roy, M., Waldo, G. \& Jennings, P.A. The rough energy landscape of superfolder GFP is linked to the chromophore. $J$ Mol Biol 373, 476-90 (2007).

29. Kozakov, D. et al. The ClusPro web server for protein-protein docking. Nat Protoc 12, 255-278 (2017).

30. Shu, X., Shaner, N.C., Yarbrough, C.A., Tsien, R.Y. \& Remington, S.J. Novel chromophores and buried charges control color in mFruits. Biochemistry 45, 9639-47 (2006).

31. Shaner, N.C. et al. Improved monomeric red, orange and yellow fluorescent proteins derived from Discosoma sp. red fluorescent protein. Nat Biotechnol 22, 1567-72 (2004). 
32. Bajar, B.T., Wang, E.S., Zhang, S., Lin, M.Z. \& Chu, J. A Guide to Fluorescent Protein FRET Pairs. Sensors (Basel) 16(2016).

33. Shcherbo, D. et al. Practical and reliable FRET/FLIM pair of fluorescent proteins. BMC Biotechnol 9, 24 (2009).

34. van der Krogt, G.N., Ogink, J., Ponsioen, B. \& Jalink, K. A comparison of donor-acceptor pairs for genetically encoded FRET sensors: application to the Epac cAMP sensor as an example. PLoS One 3, e1916 (2008).

35. CCP4. The CCP4 suite: programs for protein crystallography. Acta Crystallogr D Biol Crystallogr 50, 760-3 (1994).

36. McCoy, A.J. et al. Phaser crystallographic software. J Appl Crystallogr 40, 658-674 (2007).

37. Emsley, P. \& Cowtan, K. Coot: model-building tools for molecular graphics. Acta Crystallogr D Biol Crystallogr 60, 2126-32 (2004).

38. Murshudov, G.N., Vagin, A.A. \& Dodson, E.J. Refinement of macromolecular structures by the maximum-likelihood method. Acta Crystallogr D Biol Crystallogr 53, 240-55 (1997).

39. Fernandes, D.D. et al. Characterization of Fluorescein Arsenical Hairpin (FIAsH) as a Probe for Single-Molecule Fluorescence Spectroscopy. Sci Rep 7, 13063 (2017). 